\title{
Three-Element Filtering Antenna Array Designed by the Equivalent Circuit Approach
}

\author{
Martin Kufa, Student Member, IEEE, Zbynek Raida, Senoir Member, IEEE, and Jordi Mateu, Senior Member, IEEE
}

\begin{abstract}
The paper introduces a design methodology of a third-order three-element patch array which has no filtering parts and yet behaves like a filtering antenna (filtenna). The design approach includes shaping the frequency response of the reflection coefficient and modeling the frequency response of the normalized realized gain. In order to control the shape of these two responses, $g_{i}$ coefficients of an equivalent low-pass prototype filter are derived for designing the third-order filtering antenna array. The design methodology was verified over the frequency band from $4.8 \mathrm{GHz}$ to $6.8 \mathrm{GHz}$; for levels of the reflection coefficient from $-10 \mathrm{~dB}$ to $-20 \mathrm{~dB}$ and for fractional bandwidths from $7 \%$ to $14 \%$. The whole design methodology was supported by manufacturing and measuring three third-order filtering antenna arrays designed with different configurations. Simulated and measured results show good agreement in all cases.
\end{abstract}

Index Terms-Filtering, antenna arrays, antenna pattern synthesis.

\section{INTRODUCTION}

Today's wireless communication systems are expected to be small to provide easy mobility. The design of wireless components should be quick and easy to implement.

To obtain small dimensions of communication hardware, filtering properties of a band-pass filter can be combined with radiating properties of an antenna array. This type of device is called a filtering antenna or a filtenna. Since the filtenna plays the role of a radiator and a frequency filter at the same time, a filter at the antenna input does not need to be implemented.

In some works, the filtenna is understood as a band-pass filter with a cascaded antenna, where both have been designed separately on a common substrate [1]-[6]. This paper turns attention to the filtenna design comprising a filter synthesis approach and an antenna array approach at the same time.

A corresponding filtenna can have a structure of a bandpass filter with the last resonator replaced by a radiating element. Then, the filtenna is designed as a complete device but filtering and radiating parts can be identified [7]-[13].

In order to simplify and speed-up the design of electromagnetic devices, an equivalent circuit approach can be applied for the synthesis. The equivalent circuit approach to the synthesis of the filtering antennas has already been published in [14], [15] and [16].

In this paper, we present a novel approach to the synthesis of filtering antennas. The synthesis takes into account requirements on the frequency response of the reflection coefficient at the antenna input, and the frequency response of the realized gain in the main-lobe direction.

The frequency response of the realized gain should create an equivalent of the frequency response of the transmission coefficient of a bandpass filter. The spatial filtering (made by currents on antenna elements) and the frequency filtering (caused by resonances of antenna elements) form the final shape of the frequency response of the gain of a filtenna.

The matching objective is met by applying the equivalent circuit approach [17]-[19]. When optimizing the gain objective, the radiation pattern is calculated from the electric surface current [20].

For implementing the filtenna, we use a three-element patch array fed by apertures. The array plays the role of the filter and the antenna at the same time [17].

Section II describes the architecture of a third-order filtering antenna array fed by apertures, where each radiating element increases the order of the filtenna. In Section III, we introduce an equivalent circuit approach. In Section IV, we describe the synthesis of the filtering antenna array, and cover the following topics: (A) the synthesis of the frequency response of the reflection coefficient and adoption of the filter approach for designing the filtering array, (B) the synthesis of the frequency response of the realized gain and adoption of the antenna approach for designing the filtenna, $(\mathrm{C})$ derivation of $g_{i}$ coefficients of an equivalent low-pass prototype filter for designing the third-order filtering array fed by apertures, and (D) calculation of physical dimensions of the filtering array. In Section $\mathrm{V}$, we verify theoretical results by full-wave simulations in CST Microwave Studio. In Section VI, the manufactured three-element filtering array with different input settings is measured. Section VII concludes the paper.

\section{FILTERING ARRAY FED BY APERTURES}

The out-of-line serial feeding network of the third-order was selected as a structure for the design of the filtering array. In this case, the feeding network has a minimal effect on radiation patterns and the direction of the main lobe. The final selectivity of the gain is very good thanks to the combination of the spatial filtering and frequency filtering.

In order to minimize the influence of the feeder on radiation patterns and the main-lobe direction, elements of the

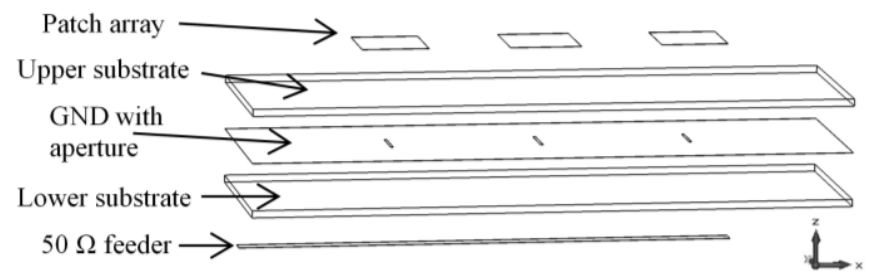

Fig. 1. Filtering patch array fed by apertures. 
antenna array are fed by a microstrip transmission line via apertures. Therefore, two substrates have to be used to build the filtenna (see Fig. 1).

Both the substrates are covered by a metallic foil on both surfaces, and the central metallic layer is shared. In this work, ARLON $25 \mathrm{~N}$ is considered (relative permittivity $\varepsilon_{r}=3.38$, and thickness $h=1.524 \mathrm{~mm}$ ).

A 50-ohm metallic feeder is created on the bottom surface, apertures are etched in the central surface, and metallic rectangular patches are located on the top surface (see Fig. 1).

\section{EQUiVALENT CIRCUIT OF FILTERING ARRAY}

Figure 2 shows an equivalent circuit of a third-order filtering patch array fed by apertures [17]. The equivalent circuit consists of three parallel $R L C$ resonant circuits, three mutual capacitances $C_{m}$, three $J$-inverters and three transmission line segments, with an additional open-ended line.

Parallel $R L C$ resonant circuits simulate the behavior of individual patches, and resistors $R$ represent radiation into the environment. Each $R L C$ increases the order of the filtenna (the third-order filtering array is obtained). Integrating current distributions on the individual patches, the radiation diagram is formed and the spatial filtering behavior of the filtenna is contributed.

Values of $J$-inverters represent coupling between the feeder and patches, and values of $C_{m}$ simulate the mutual capacitance between the feeder and patches.

The width of the microstrip feeder is selected to obtain 50-ohm characteristic impedance. The length of the first to the third segment is the wavelength and the length of the openended segment is a quarter of the wavelength, approximately.

Using the equivalent circuit approach for the synthesis of the filtenna, a significant role is played by the mutual capacitance and the coupling between the microstrip feeder and patches. Both the mutual capacitances and the couplings are essentially defined by the width of apertures $W_{s}$. Therefore, the effect of the width of the aperture $W_{s}$ on the mutual capacitance and the coupling has to be described accurately.

The mutual capacitance and the value of the $J$-inverter can be obtained for a single patch fed via an aperture by a 50-ohm open-ended transmission line. The capacitances and

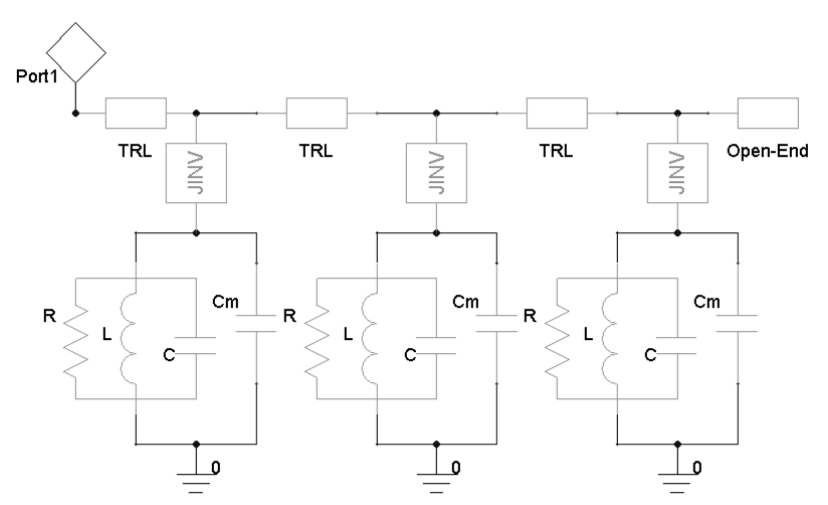

Fig. 2. Equivalent circuit of a three-element filtering array.

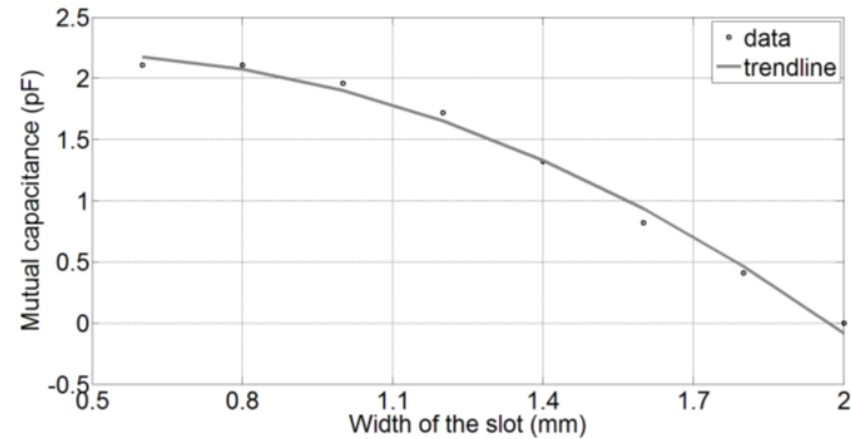

Fig. 3. Influence of the width of the aperture $W_{s}$ on mutual capacitance between the patch and the feeder at the frequency $5.8 \mathrm{GHz}$ for ARLON $25 \mathrm{~N}\left(\varepsilon_{r}=3.38\right.$ and $\left.h=1.524 \mathrm{~mm}\right)$.

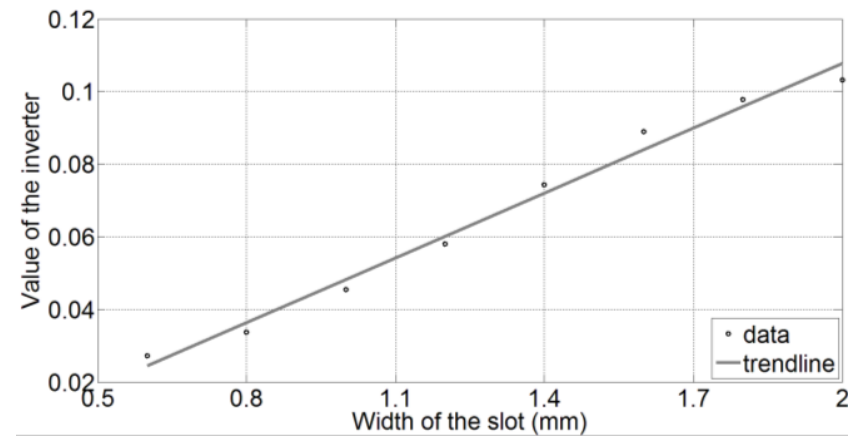

Fig. 4. Influence of the width of the aperture $W_{s}$ on $J$-inverter value simulating coupling between the patch and the feeder at the frequency $5.8 \mathrm{GHz}$ for ALON $25 \mathrm{~N}\left(\varepsilon_{r}=3.38\right.$ and $\left.h=1.524 \mathrm{~mm}\right)$.

$J$-inverter values can be computed using a suitable full-wave simulator [18] or comparing the behavior of an equivalent model with a corresponding response of a full-wave model.

Dependencies of mutual capacitances and $J$-inverter values on the width of the aperture $W_{s}$ have been researched at $5.8 \mathrm{GHz}$ for ARLON $25 \mathrm{~N}\left(h=1.524 \mathrm{~mm}\right.$, and $\left.\varepsilon_{r}=3.38\right)$. The dependencies are going to be included into the synthesis of the frequency response of reflection coefficient in Section IV.

Dependency of the mutual capacitance on the width of the aperture $W_{s}$ is shown in Fig. 3, and dependency of the $J$-inverter value on the aperture width $W_{s}$ is depicted in Fig. 4. With respect to easier future implementation, the aperture width $W_{s}$ is related to the width of the patch $W_{a}$ which corresponds to the desired frequency.

$$
\begin{gathered}
C_{m}=-1.640 \cdot 10^{2} \cdot W_{N}^{2}+1.070 \cdot 10^{1} \cdot W_{N}+2.026 \\
J=7.894 \cdot 10^{-1} \cdot W_{N}-1.115 \cdot 10^{-2}
\end{gathered}
$$

In (1) and (2), $W_{N}=W_{s} / W_{a}$ denotes this normalized value.

If the equivalent circuit in Fig. 2 is understood as a conventional frequency filter, the open end plays the role of the output port. Since energy radiated by patches (represented by $R L C$ resonators) is consumed by particular resistors $R$, the signal at the open end should be minimal.

For spatial filtering, amplitudes and phases of currents on patches ( $R L C$ circuits) are dominant. Therefore, the requested spatial filtering can be obtained by the optimization of the 
equivalent circuit for a requested amplitude and phase distribution over $R L C$ circuits.

\section{SYNTHESIS OF FILTERING ARRAY}

In this Section, we derive formulas for the synthesis of the frequency response of the reflection coefficient at the input of the filtering array, and for the synthesis of the frequency response of the realized gain in the main-lobe direction.

The frequency response of the realized gain [21]

$$
\left|S_{21 R G}\right|=D \cdot A F \cdot\left(1-\left|S_{11}\right|^{2}\right)
$$

can be understood as an equivalent of the frequency response of the transmission coefficient (the wave passing the output port is replaced by the radiation of electromagnetic energy to the main-lobe direction).

In (3), $D$ is directivity of a single patch, $A F$ denotes array factor and $S_{11}$ is reflection coefficient at the antenna input.

Equation (3) can be rewritten to [21]

$$
\left|S_{21 R G}\right|=D \cdot A F \cdot\left|S_{21}^{\prime}\right|^{2},
$$

where $\mathrm{S}_{21}{ }^{\prime}$ corresponds to losses in dielectrics and losses by radiation.

When synthesizing a filter, $g_{i}$ coefficients of the low-pass prototype can shape the frequency response of the reflection coefficient $S_{11}$ and the transmission coefficient $S_{21}$ '. Obviously, we can shape the frequency response of the realized gain $S_{21 R G}$ in a similar way.

Since conventional approximations of filter characteristics (Chebyshev, Butterworth, etc.) are not applicable in the case of filtering arrays, alternative coefficients are going to be derived in the next Section.

\section{A. Synthesis of Frequency Response of Reflection Coefficient}

In order to analyze the third-order filtering array of patches (the combination of equivalent $R L C$ circuits) resonating at different frequencies $f_{0 a}(i)$, we use the theory of asynchronously tuned filters [18]

$$
f_{0 a}(i)=f_{0} \pm \frac{F B W \cdot f_{0}}{2} .
$$

$F B W$ is the fractional bandwidth between resonant frequencies $f_{0 a}(i)$, and $f_{0}$ is the center frequency of the filtering array.

In the third-order filtenna, the first patch and the third patch (equivalent $R L C$ circuits) are tuned to the upper resonant frequency. The second patch (an equivalent $R L C$ circuit) is tuned to the lower resonant frequency.

Capacitances and inductances of parallel resonant circuits representing patches in the array can be evaluated by [18]

$$
\begin{gathered}
C(i)=\frac{1}{2 \cdot \pi \cdot f_{0 a}(i) \cdot R \cdot F B W_{a}}, \\
L(i)=\frac{1}{\left[2 \cdot \pi \cdot f_{0 a}(i)\right]^{2} \cdot C(i)} .
\end{gathered}
$$

For evaluating $J$-inverters among individual patches and the feeder, we can use [18]

$$
J(i)=\sqrt{\frac{Y_{0} \cdot C(i) \cdot F B W \cdot 2 \cdot \pi \cdot f_{0}}{\Omega_{C} \cdot g_{0} \cdot g_{i}}} .
$$

From the value of the $J$-inverters (8) and the dependencies of the mutual capacitance and coupling on the width of the aperture $W_{s}$, we can calculate mutual capacitances between the patch and feeder by using (1) and (2)

$$
\begin{aligned}
& C_{m}(i)=-1.640 \cdot 10^{2} \cdot\left(\frac{J(i)+1.115 \cdot 10^{-2}}{7.894 \cdot 10^{-1}}\right)^{2}+\ldots . \\
& +1.070 \cdot 10^{1} \cdot \frac{J(i)+1.115 \cdot 10^{-2}}{7.894 \cdot 10^{-1}}+2.026
\end{aligned}
$$

The resistance $R$ in the equivalent circuit denotes the value of the resistor which simulates the antenna radiation. Next, $C(i)$ and $L(i)$ are individual capacitances and inductances representing patches. Finally, $C_{m}(i)$ is the mutual capacitance between the patch and the feeder (see Fig. 2). The value of the fractional frequency bandwidth of a single patch fed by an aperture is denoted as $F B W_{a}, Y_{0}$ represents the characteristic admittance of the microstrip feeder, $g_{0}$ and $g_{i}$ are coefficients of the third-order low-pass prototype filter and $\Omega_{C}$ is the cutoff angular frequency of the filter.

The lossless transmission lines between adjacent parallel resonant circuits are modeled by $A B C D$ matrices [18]

$$
\left[\begin{array}{ll}
A & B \\
C & D
\end{array}\right]=\left[\begin{array}{cc}
\cos (\beta \cdot l) & j \cdot Z_{c} \cdot \sin (\beta \cdot l) \\
j \cdot Y_{c} \cdot \sin (\beta \cdot l) & \cos (\beta \cdot l)
\end{array}\right]
$$

The line is described by the characteristic impedance (admittance) $Z_{c}\left(Y_{c}\right)$, the length $l$ and the phase constant $\beta$.

The $J$-inverter can be described by the $A B C D$ matrix [18]

$$
\left[\begin{array}{ll}
A & B \\
C & D
\end{array}\right]=\left[\begin{array}{cc}
0 & \pm \frac{1}{j \cdot J} \\
\mp j \cdot J & 0
\end{array}\right] .
$$

Using the matrix for general impedance [18], the $A B C D$ matrix of the parallel $R L C$ circuit is obtained

$$
\left[\begin{array}{ll}
A & B \\
C & D
\end{array}\right]=\left[\begin{array}{cc}
1 & j \cdot \omega \cdot R \cdot L \\
0 & R-\omega^{2} \cdot R \cdot L \cdot C+j \cdot \omega \cdot L
\end{array}\right] .
$$

The filtering antenna array fed by apertures can be quickly designed by a script implementing equations (10) to (12).

Using equations (11), (12) and considering the condition for the short end of a one-port network [18], [19], the $A B C D$ matrix for a parallel combination of a resonant circuit and a $J$-inverter can be obtained

$$
\left[\begin{array}{ll}
A & B \\
C & D
\end{array}\right]=\left[\begin{array}{cc}
1 & 0 \\
\frac{j \cdot \omega \cdot R \cdot L \cdot J^{2}}{R-\omega^{2} \cdot R \cdot L \cdot C+j \cdot \omega \cdot L} & 1
\end{array}\right] .
$$

Next, the $A B C D$ matrix of the equivalent circuit of the whole antenna structure is calculated by multiplying (10) and (13):

$$
\begin{aligned}
& T O T A L=M T L_{s} \cdot M P B_{1} \cdot M T L_{1} \cdot \ldots . \\
& \cdot M P B_{2} \cdot M T L_{2} \cdot M P B_{3} \cdot M T L_{o}
\end{aligned}
$$

Here, $M T L_{s}$ is the $A B C D$ matrix of the line from the source to the first patch and $M T L_{o}$ is the $A B C D$ matrix of the line from the last patch to the open end of the line. $M T L_{1}$ is the $A B C D$ 
matrix of the line between the first patch and the second patch, and $M T L_{2}$ is the $A B C D$ matrix of the line between the second patch and the third patch. The $A B C D$ matrices $M T L_{1}$ and $M T L_{2}$ dominantly influence the main-lobe direction and the phase feeding of the patch antennas. $M P B_{1}, M P B_{2}$ and $M P B_{3}$ are $A B C D$ matrices of parallel branches (patches).

The reflection coefficient of the equivalent circuit may be calculated from the total $A B C D$ matrix (14) using [18], [19]

$$
S_{11}=\frac{A+B \cdot Y_{0}-C \cdot Z_{0}-D}{A+B \cdot Y_{0}+C \cdot Z_{0}+D}
$$

\section{B. Synthesis of Frequency Response of Realized Gain}

In order to calculate the frequency response of the normalized realized gain of the filtering antenna, we have to consider [20]

$$
\begin{aligned}
& \left|E_{\Theta}(\Theta)\right|^{2}=\varepsilon_{r e} \cdot\left[1+\varepsilon_{r} \cdot \cot ^{2}\left(k_{0} \cdot h \cdot \sqrt{\varepsilon_{r}}\right)\right] \frac{\cos ^{2}\left(k_{0} \cdot L_{a} \cdot \sin \Theta / 2\right)}{\left(\varepsilon_{r e}-\sin ^{2} \Theta\right)^{2}} \ldots \\
& \frac{\left(\varepsilon_{r e}-\sin ^{2} \Theta\right) \cdot \cos ^{2} \Theta}{\left(\varepsilon_{r e}-\sin ^{2} \Theta\right)+\varepsilon_{r}^{2} \cdot \cos ^{2} \Theta \cdot \cot ^{2}\left(k_{0} \cdot h \cdot \sqrt{\varepsilon_{r}-\sin ^{2} \Theta}\right)}
\end{aligned}
$$

to describe the radiation pattern of a patch in the E-plane, and [20]

$$
\begin{aligned}
& \left|E_{\Phi}(\Theta)\right|^{2}=\left[1+\varepsilon_{r} \cdot \cot ^{2}\left(k_{0} \cdot h \cdot \sqrt{\varepsilon_{r}}\right)\right] \sin c^{2}\left(k_{0} \cdot W_{a} \cdot \sin \cdot \Theta / 2\right) \ldots \\
& \frac{\cos ^{2} \Theta}{\left(\varepsilon_{r}-\sin ^{2} \Theta\right) \cot ^{2}\left(k_{0} \cdot h \cdot \sqrt{\varepsilon_{r}-\sin ^{2} \Theta}\right)+\cos ^{2} \Theta}
\end{aligned}
$$

to describe the radiation pattern of a patch in the H-plane.

Here, $\varepsilon_{r}$ denotes relative permittivity of the substrate, $h$ is the thickness of the substrate, $\varepsilon_{r e}$ is the effective dielectric constant, $k_{0}$ is the free-space wave number, $L_{a}$ is the length of the patch and $W_{a}$ is the width of the patch. [20]

Relations (16) and (17) can be used to express directivity

$$
D=\frac{4 \cdot \pi \cdot r^{2}\left(\left|E_{\Theta}\right|^{2}+\left|E_{\Phi}\right|^{2}\right)_{\Theta=0}}{2 \cdot \eta_{0} \cdot P_{r}} .
$$

Here, $r$ is the distance of the observation point from the patch antenna; $E_{\Theta}$ and $E_{\Phi}$ are radiation patterns of the patch antenna in the E- and H-plane, respectively. Next, $\eta_{0}$ is wave impedance of free space and $P_{r}$ is the radiated power.

In order to calculate the total radiation pattern of the filtering array represented by the equivalent circuit (Fig. 1 and Fig. 2) [17], an array factor has to be defined [22], [23]

$$
A F=\frac{\sin (N \cdot \Psi / 2)}{\sin (\Psi / 2)}
$$

where $N$ is the number of radiation elements and

$$
\Psi=k_{0} \cdot d \cdot \cos \Theta+\xi \cdot
$$

In (20), $k_{0}$ is the free-space wave number, $d$ is the distance between two patches and $\xi$ describes the phase shift between two patches.

In order to obtain the frequency response of the realized gain, the frequency response of the reflection coefficient [17] has to be calculated.

\section{Three-Element Filtering Array and $g_{i}$ Coefficients}

In this section, new $g_{i}$ coefficients for a third-order threeelement filtering array fed by apertures are defined. These $g_{i}$ coefficients enable us to control frequency responses of the reflection coefficient and the realized gain. Coefficients are computed for a specific value of the reflection coefficient and an acceptable value of the fractional bandwidth of the array.

The $g_{i}$ coefficients are obtained by optimizing the shape of frequency responses of the reflection coefficient and the realized gain by a script implementing equations given above.

For an acceptable reflection coefficient $S_{11}<-10 \mathrm{~dB}$, the relation between the fractional bandwidth $F B W_{s}$ of the whole structure and $F B W$ between resonant frequencies of patches is

$$
F B W=\frac{-5.598 \cdot 10^{-1}+\sqrt{\left(5.598 \cdot 10^{-1}\right)^{2}-4 \cdot 6.303 \cdot\left(6.809 \cdot 10^{-2}-F B W_{s}\right)}}{2 \cdot 6.303} .
$$

Here, $F B W_{s}$ presents the fractional bandwidth of the realized gain for a decrease of $3 \mathrm{~dB}$. In this case, the fractional bandwidth $F B W_{s}$ can be set within the interval from $7 \%$ to $14 \%$. Equation (21) describes the dependence of the fractional bandwidth of the whole structure $F B W_{s}$ on the fractional bandwidth between resonant frequencies of patches $F B W$.

Now, the value of $F B W$ can be used for evaluating $g_{i}$ coefficients

$$
\begin{gathered}
g_{0}=g_{4}=1, \\
g_{1}=g_{3}=-4.778 \cdot 10^{2} \cdot F B W^{2}+5.506 \cdot 10^{1} \cdot F B W-5.333 \cdot 10^{-2}, \\
g_{2}=-5.097 \cdot 10^{2} \cdot F B W^{2}+5.536 \cdot 10^{1} \cdot F B W+1.533 \cdot 10^{-1} .
\end{gathered}
$$

In (22) to (24), the values of $g_{0}$ and $g_{4}$ represent normalized impedances of the feeder and the open-ended transmission line. Coefficients $g_{1}$ and $g_{3}$ can influence the final value of the first $J$-inverter and the third $J$-inverter (frequency response of $S_{11}$ and $S_{21 R G}$ ). The coefficient $g_{2}$ represents the final value of the second $J$-inverter, and the frequency response of $S_{11}$ and $S_{21 R G}$, respectively.

Equations (21) to (24) have to be recalculated if the requested match should be:

A) Better than $S_{11}<-15 \mathrm{~dB}$,

$$
\begin{gathered}
F B W=\frac{-5.495 \cdot 10^{-1}+\sqrt{\left(5.495 \cdot 10^{-1}\right)^{2}-4 \cdot 6.261 \cdot\left(7.445 \cdot 10^{-2}-F B W_{s}\right)}}{2 \cdot 6.261}, \\
g_{1}=g_{3}=-3.727 \cdot 10^{2} \cdot F B W^{2}+4.402 \cdot 10^{1} \cdot F B W-5.733 \cdot 10^{-2}, \\
g_{2}=-3.329 \cdot 10^{2} \cdot F B W^{2}+4.149 \cdot 10^{1} \cdot F B W+2.510 \cdot 10^{-1} .
\end{gathered}
$$

B) Better than $S_{11}<-20 \mathrm{~dB}$

$$
\begin{gathered}
F B W=\frac{-5.209 \cdot 10^{-1}+\sqrt{\left(5.209 \cdot 10^{-1}\right)^{2}-4 \cdot 6.344 \cdot\left(7.942 \cdot 10^{-2}-F B W_{s}\right)}}{2 \cdot 6.344}, \\
g_{1}=g_{3}=-2.835 \cdot 10^{2} \cdot F B W^{2}+3.592 \cdot 10^{1} \cdot F B W-5.333 \cdot 10^{-3}, \\
g_{2}=-4.484 \cdot 10^{2} \cdot F B W^{2}+5.044 \cdot 10^{1} \cdot F B W-1.117 \cdot 10^{-2} .
\end{gathered}
$$


C) Better than $S_{11}<-25 \mathrm{~dB}$

$$
\begin{gathered}
F B W=\frac{7.001 \cdot 10^{-1}+\sqrt{\left(7.001 \cdot 10^{-1}\right)^{2}-4 \cdot 2.013 \cdot 10^{1} \cdot\left(1.070 \cdot 10^{-1}-F B W_{s}\right)}}{2 \cdot 2.013 \cdot 10^{1}}, \\
g_{1}=g_{3}=-2.753 \cdot 10^{1} \cdot F B W^{2}+1.207 \cdot 10^{1} \cdot F B W+4.363 \cdot 10^{-1}, \\
g_{2}=-2.566 \cdot 10^{2} \cdot F B W^{2}+3.225 \cdot 10^{1} \cdot F B W+3.460 \cdot 10^{-1} .
\end{gathered}
$$

\section{Dimensions of Filtenna Model}

The width $W_{a}(i)$ and the length $L_{a}(i)$ of patches are [23]

$$
\begin{gathered}
W_{a}(i)=\frac{c}{2 \cdot f_{0 a}(i) \cdot \sqrt{\varepsilon_{r}}}, \\
L_{a}(i)=\frac{c}{2 \cdot f_{0 a}(i)_{0} \cdot \sqrt{\varepsilon_{r e}}}-2 \cdot \Delta .
\end{gathered}
$$

Here, $\Delta$ represents fringing fields, $c$ is the speed of light in free space, $f_{0}(i)$ is the asynchronously tuned resonant frequency (each patch antenna is tuned at a different frequency), $\varepsilon_{r}$ is the relative permittivity, $\varepsilon_{r e}$ is the effective dielectric constant and $h$ is the thickness of the substrate.

Considering outputs of Section II, the width of apertures can be calculated using

$$
W_{s}(i)=\frac{c \cdot J(i)+1.115 \cdot 10^{-2}}{7.894 \cdot 10^{-1} \cdot 2 \cdot f_{0 a}(i) \cdot \sqrt{\varepsilon_{r}}} .
$$

The length of the apertures is

$$
L_{s}(i)=\frac{0.572 \cdot c}{2 \cdot f_{0 a}(i) \cdot \sqrt{\varepsilon_{r}}} .
$$

Other parameters (the width of the feeder, the distance between two neighboring patches and the length of the open end of the feeder) can be calculated following [18] and [19].

\section{THEORETICAL RESUltS VERSUS FULL-WAVE RESUltS}

In this section, the methodology of the design of the filtering array is verified. Parameters of the designed filtenna are evaluated by a full-wave simulation in CST Microwave Studio initially. The numerical study comprises different values of parameters; $f_{0}, F B W$ and $S_{11}$.

Parameters $f_{0}, F B W$ and $S_{11}$ obtained by the full-wave simulation are compared with the equivalent circuit approach. The comparison does not consider a connector, losses in the dielectrics and adhesives between substrates.

For specified requirements, parameters of the equivalent circuit have been computed (the left column in Table I). Using ARLON $25 \mathrm{~N}\left(h=1.524 \mathrm{~mm}, \varepsilon_{r}=3.38\right)$, dimensions of the antenna can be obtained (the central column in Table I). In order to improve results, a built-in CST optimizer was used to improve the design (the right column in Table I).

In Fig. 5, frequency responses of the reflection coefficient at the filtenna input $S_{11}$ and transmission coefficient (normalized realized gain $R G$ ) are depicted. Here, MATLAB represents the equivalent circuit, CST represents full-wave simulation of the equivalent planar implementation, and CST opt. is the optimized planar implementation. Obviously, all frequency responses agree sufficiently.
TABLE I. VALUES OF ELEMENTS IN THE EQUIVALENT CIRCUIT OF THE FILTENNA (LEFT), DIMENSIONS OF PLANAR IMPLEMENTATION OF THE FILTENNA (CENTER) AND DIMENSIONS OF THE OPTIMIZED FILTENNA (RIGHT); $f_{0}=5.8 \mathrm{GHz} ; F B W=13 \%$ AND $S_{11}<-15 \mathrm{DB}$.

\begin{tabular}{lrlrll}
\hline \multicolumn{2}{c}{ Equivalent circuit } & \multicolumn{2}{c}{ Dimensions from script } & \multicolumn{2}{c}{ CST optimized } \\
\hline$R_{1}, R_{3}[\Omega]$ & 50.37 & $W_{a 1}, W_{a 3}[\mathrm{~mm}]$ & 12.42 & $W_{a 1}, W_{a 3}[\mathrm{~mm}]$ & 12.21 \\
$R_{2}[\Omega]$ & 50.37 & $W_{a 2}[\mathrm{~mm}]$ & 13.18 & $W_{a 2}[\mathrm{~mm}]$ & 12.96 \\
$L_{1}, L_{3}[\mathrm{pH}]$ & 61.00 & $L_{a 1}, L_{a 3}[\mathrm{~mm}]$ & 11.88 & $L_{a 1}, L_{a 3}[\mathrm{~mm}]$ & 11.68 \\
$L_{2}[\mathrm{pH}]$ & 64.77 & $L_{a 2}[\mathrm{~mm}]$ & 12.62 & $L_{a 2}[\mathrm{~mm}]$ & 12.40 \\
$C_{1}, C_{3}[\mathrm{pF}]$ & 9.62 & $W_{s 1}, W_{s 3}[\mathrm{~mm}]$ & 0.48 & $W_{s 1}, W_{s 3}[\mathrm{~mm}]$ & 0.47 \\
$C_{2}[\mathrm{pF}]$ & 10.21 & $W_{s 2}[\mathrm{~mm}]$ & 0.48 & $W_{s 2}[\mathrm{~mm}]$ & 0.46 \\
$J_{1}, J_{3}[\mathrm{mS}]$ & 19.30 & $L_{s 1}, L_{s 3}[\mathrm{~mm}]$ & 7.11 & $L_{s 1}, L_{s 3}[\mathrm{~mm}]$ & 7.12 \\
$J_{2}[\mathrm{mS}]$ & 17.90 & $L_{s 2}[\mathrm{~mm}]$ & 7.55 & $L_{s 2}[\mathrm{~mm}]$ & 7.32 \\
$w[\mathrm{~mm}]$ & 3.30 & $w[\mathrm{~mm}]$ & 3.30 & $w[\mathrm{~mm}]$ & 3.30 \\
$d[\mathrm{~mm}]$ & 27.10 & $d[\mathrm{~mm}]$ & 27.10 & $d[\mathrm{~mm}]$ & 28.6 \\
$l_{\mathrm{o}}[\mathrm{mm}]$ & 8.00 & $l_{\mathrm{o}}[\mathrm{mm}]$ & 8.00 & $l_{\mathrm{o}}[\mathrm{mm}]$ & 7.43 \\
\hline
\end{tabular}

TABLE II. COMPARISON OF RESONANT FREQUENCY, FRACTIONAL BANDWIDTH AND REFLECTION COEFFICIENT COMPUTED FOR THE EQUIVALENT CIRCUIT (LEFT), PLANAR IMPLEMENTATION (CENTER) AND OPTIMIZED IMPLEMENTATION (RIGHT); $f_{0}=5.8 \mathrm{GHz} ; F B W=13 \%$ AND $S_{11}<-15 \mathrm{DB}$.

\begin{tabular}{lrrr}
\hline & Script & CST 1 $^{\text {st }}$ run & CST opt. \\
\hline$f_{0}[\mathrm{GHz}]$ & 5.77 & 5.68 & 5.79 \\
$F B W_{-3 \mathrm{~dB}}[\%]$ & 14.91 & 14.85 & 14.37 \\
$S_{11}<[\mathrm{dB}] @ f_{0}$ & -16.79 & -14.34 & -16.53 \\
$F B W_{-10 \mathrm{~dB}}[\%]$ & 8.62 & 9.68 & 8.96 \\
$S_{21}$ suppression $[\mathrm{dB}]$ & -17.53 & -17.95 & -19.08 \\
\hline
\end{tabular}

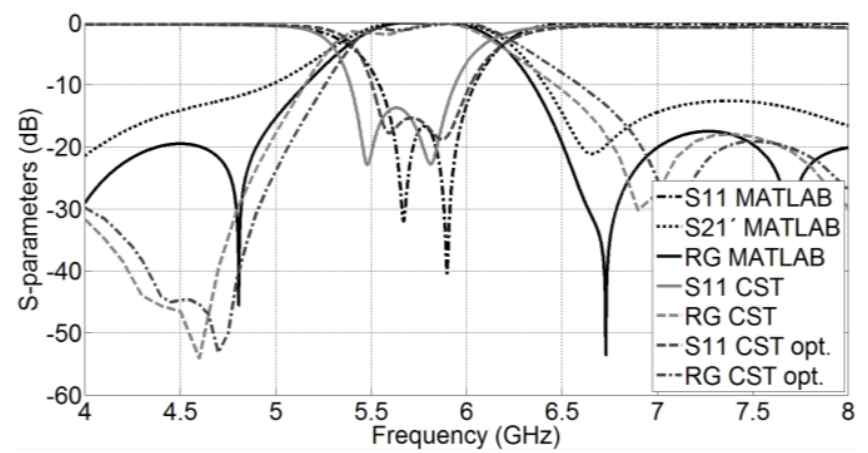

Fig. 5. Frequency responses of reflection coefficient $S_{11}$, transmission coefficient $S_{21}$ (or normalized realized gain $R G$ ) for the equivalent circuit (MATLAB), planar implementation (CST) and optimized planar implementation (CST opt.); $f_{0}=5.8 \mathrm{GHz} ; F B W=13 \%$ and $S_{11}<-15 \mathrm{~dB}$.

Next, numerical values of the resonant frequency $f_{0}$, fractional bandwidth $F W B$ and the magnitude of reflection coefficient $S_{11}$ at the resonant frequency are compared (see Table II). We can see a reasonable match here.

In the second testing example, the filtenna was designed for the center frequency $6.8 \mathrm{GHz}$; the fractional bandwidth $10 \%$ and the reflection coefficient better than $-10 \mathrm{~dB}$.

Table III summarizes component values of the equivalent circuit model (left), dimensions of planar implementation (center) and optimized implementation (right).

In Fig. 6, frequency responses of reflection and transmission coefficients of the equivalent circuit model 
TABLE III. VALUES OF ELEMENTS IN THE EQUIVALENT CIRCUIT OF THE FILTENNA (LEFT), DIMENSIONS OF PLANAR IMPLEMENTATION OF THE FILTENNA (CENTER) AND DIMENSIONS OF THE OPTIMIZED FILTENNA (RIGHT); $f_{0}=6.8 \mathrm{GHz} ; F B W=10 \%$ AND $S_{11}<-10 \mathrm{DB}$.

\begin{tabular}{lrl|rlc}
\hline \multicolumn{2}{c}{ Equivalent circuit } & \multicolumn{2}{l}{ Dimensions from script } & \multicolumn{2}{c}{ CST optimized } \\
\hline$R_{1}, R_{3}[\Omega]$ & 50.37 & $W_{a 1}, W_{a 3}[\mathrm{~mm}]$ & 10.70 & $W_{a 1}, W_{a 3}[\mathrm{~mm}]$ & 10.54 \\
$R_{2}[\Omega]$ & 50.37 & $W_{a 2}[\mathrm{~mm}]$ & 11.13 & $W_{a 2}[\mathrm{~mm}]$ & 10.97 \\
$L_{1}, L_{3}[\mathrm{pH}]$ & 52.55 & $L_{a 1}, L_{a 3}[\mathrm{~mm}]$ & 10.24 & $L_{a 1}, L_{a 3}[\mathrm{~mm}]$ & 10.09 \\
$L_{2}[\mathrm{pH}]$ & 54.67 & $L_{a 2}[\mathrm{~mm}]$ & 10.65 & $L_{a 2}[\mathrm{~mm}]$ & 10.50 \\
$C_{1}, C_{3}[\mathrm{pF}]$ & 8.29 & $W_{s 1}, W_{s 3}[\mathrm{~mm}]$ & 0.35 & $W_{s 1}, W_{s 3}[\mathrm{~mm}]$ & 0.34 \\
$C_{2}[\mathrm{pF}]$ & 8.62 & $W_{s 2}[\mathrm{~mm}]$ & 0.36 & $W_{s 2}[\mathrm{~mm}]$ & 0.37 \\
$J_{1}, J_{3}[\mathrm{mS}]$ & 14.90 & $L_{s 1}, L_{s 3}[\mathrm{~mm}]$ & 6.12 & $L_{s 1}, L_{s 3}[\mathrm{~mm}]$ & 6.29 \\
$J_{2}[\mathrm{mS}]$ & 14.40 & $L_{s 2}[\mathrm{~mm}]$ & 6.37 & $L_{s 2}[\mathrm{~mm}]$ & 6.24 \\
$w[\mathrm{~mm}]$ & 3.30 & $w[\mathrm{~mm}]$ & 3.30 & $w[\mathrm{~mm}]$ & 3.30 \\
$d[\mathrm{~mm}]$ & 23.40 & $d[\mathrm{~mm}]$ & 23.40 & $d[\mathrm{~mm}]$ & 25.50 \\
$l_{\mathrm{o}}[\mathrm{mm}]$ & 6.90 & $l_{0}[\mathrm{~mm}]$ & 6.90 & $l_{\mathrm{o}}[\mathrm{mm}]$ & 6.67 \\
\hline
\end{tabular}

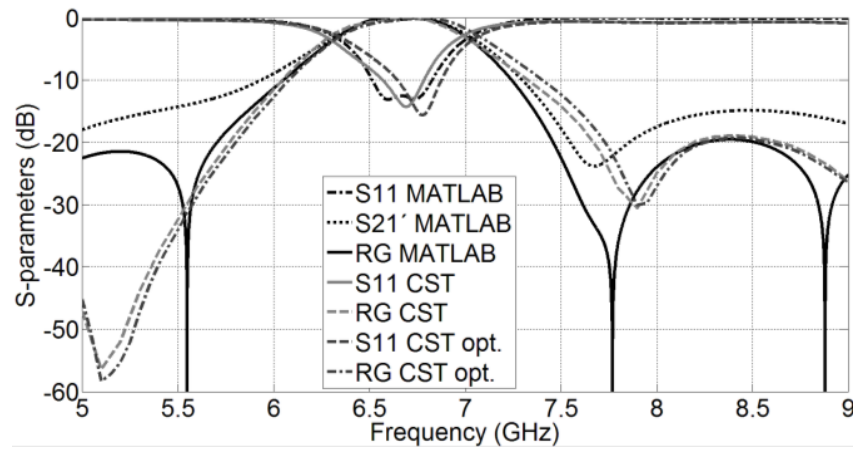

Fig. 6. Frequency responses of reflection coefficient $S_{11}$, transmission coefficient $S_{21}$ (or normalized realized gain $R G$ ) for the equivalent circuit

(MATLAB), planar implementation (CST) and optimized planar implementation (CST opt.);

$f_{0}=6.8 \mathrm{GHz} ; F B W=10 \%$ and $S_{11}<-10 \mathrm{~dB}$.

TABLE IV. COMPARISON OF RESONANT FREQUENCY, FRACTIONAL BANDWIDTH AND REFLECTION COEFFICIENT COMPUTED FOR THE EQUIVALENT CIRCUIT (LEFT), PLANAR IMPLEMENTATION (CENTER) AND OPTIMIZED IMPLEMENTATION (RIGHT); $f_{0}=6.8 \mathrm{GHz} ; F B W=10 \%$ AND $S_{11}<-10 \mathrm{DB}$

\begin{tabular}{lrrr}
\hline & Script & CST 1 $^{\text {st }}$ run & CST opt. \\
\hline$f_{0}[\mathrm{GHz}]$ & 6.67 & 6.62 & 6.69 \\
$F B W_{-3 \mathrm{~dB}}[\%]$ & 10.62 & 10.76 & 10.88 \\
$S_{11}<[\mathrm{dB}] @ f_{0}$ & -12.52 & -11.85 & -11.80 \\
$F B W_{-10 \mathrm{~dB}}[\%]$ & 4.66 & 3.18 & 3.37 \\
$S_{21}$ suppression $[\mathrm{dB}]$ & -19.57 & -18.86 & -19.27 \\
\hline
\end{tabular}

(obtained by the script in MATLAB), planar implementation (CST) and optimized implementation (CST opt.) show good agreement. The most important corresponding parameters from the comparison in Fig. 6 are given in Table IV.

\section{EXPERIMENTAL VERIFICATION}

Prototypes of filtennas specified in Table I and Table III were manufactured and measured:
TABLE V COMPARISON OF THE SIMULATED AND MEASURED RESONANT FREQUENCY, FRACTIONAL BANDWIDTH AND REFLECTION COEFFICIENT; $f_{0}=5.8 \mathrm{GHZ} ; F B W=13 \%$ AND $S_{11}<-15 \mathrm{DB}$

\begin{tabular}{lrc}
\hline & Simulation & Measurement \\
\hline$f_{0}[\mathrm{GHz}]$ & 5.86 & 5.77 \\
$F B W_{-3 \mathrm{~dB}}[\%]$ & 14.00 & 14.89 \\
$S_{11}<[\mathrm{dB}] @ f_{0}$ & -11.88 & -11.85 \\
$F B W_{-10 \mathrm{~dB}}[\%]$ & 11.02 & 10.01 \\
$S_{21}$ suppression $[\mathrm{dB}]$ & -18.01 & -21.92 \\
Realized gain $[\mathrm{dB}]$ & 8.84 & 9.13 \\
\hline
\end{tabular}

Measured and simulated parameters of Prototype I $\left(f_{0}=5.8 \mathrm{GHz}, F B W_{-3 \mathrm{~dB}}=13 \%, S_{11}<-15 \mathrm{~dB}\right)$ are given in Table V.

Simulated and measured frequency responses of the reflection coefficient and the normalized realized gain are depicted in Fig. 7. Simulated and measured radiation patterns in the E-plane (co- and cross-polarization components) are shown in Fig. 8; the plane corresponds with the $\mathrm{x}-\mathrm{z}$ plane in Fig. 1. The deviation in the main lobe direction from the normal to the substrate is shown in Fig. 9; the maximum deviation is $6.2^{\circ}$ for simulation and $5^{\circ}$ for measurement.

Measured and simulated parameters of Prototype II $\left(f_{0}=6.8 \mathrm{GHz}, F B W_{-3 \mathrm{~dB}}=10 \%, S_{11}<-10 \mathrm{~dB}\right)$ are given in Table VI.

Simulated and measured frequency responses of the reflection coefficient and the normalized realized gain are depicted in Fig. 10. Simulated and measured radiation patterns

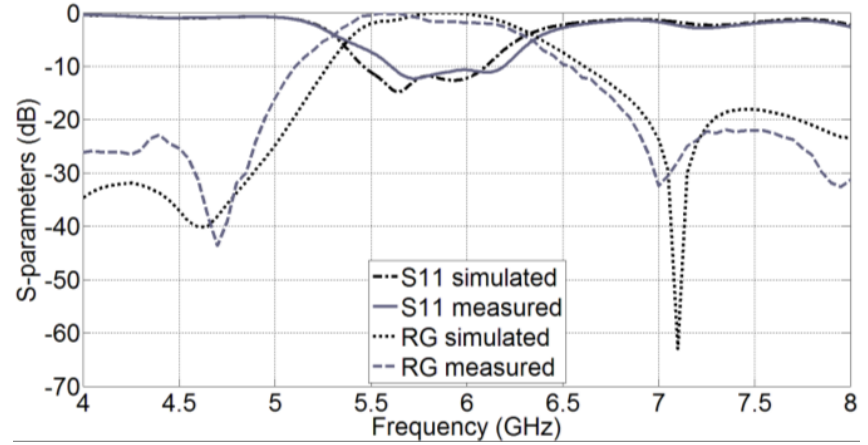

Fig. 7. Comparison of simulated and measured frequency responses of reflection coefficient $S_{11}$ and normalized realized gain RG $f_{0}=5.8 \mathrm{GHz} ; F B W=13 \%$ and $S_{11}<-15 \mathrm{~dB}$.

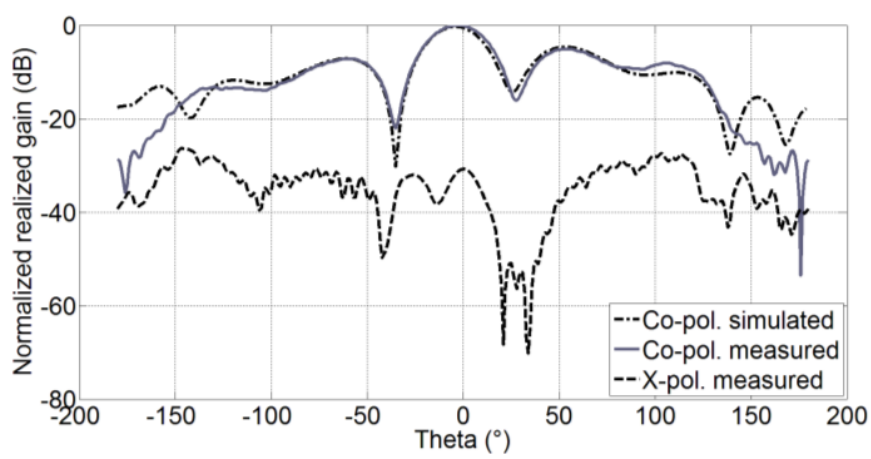

Fig. 8. Comparison of simulated and measured radiation patterns (co-polar and cross-polar components) in the E-plane at frequency $5.8 \mathrm{GHz}$ 


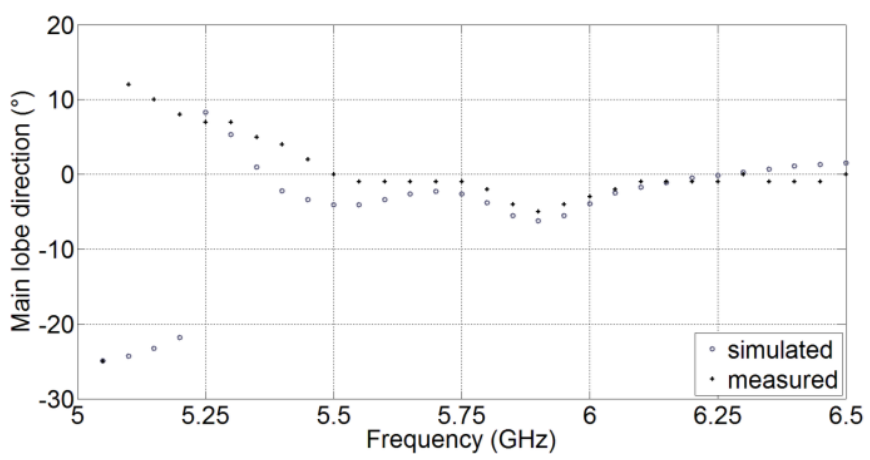

Fig. 9. Comparison of the simulated and measured frequency response of the main lobe direction of the filtenna over the operation band; $f_{0}=5.8 \mathrm{GHz} ; F B W=13 \%$ and $S_{11}<-15 \mathrm{~dB}$.

TABLE VI. COMPARISON OF THE SIMULATED AND MEASURED RESONANT FREQUENCY, FRACTIONAL BANDWIDTH AND REFLECTION COEFFICIENT; $f_{0}=6.8 \mathrm{GHZ} ; F B W=10 \%$ AND $S_{11}<-10 \mathrm{DB}$.

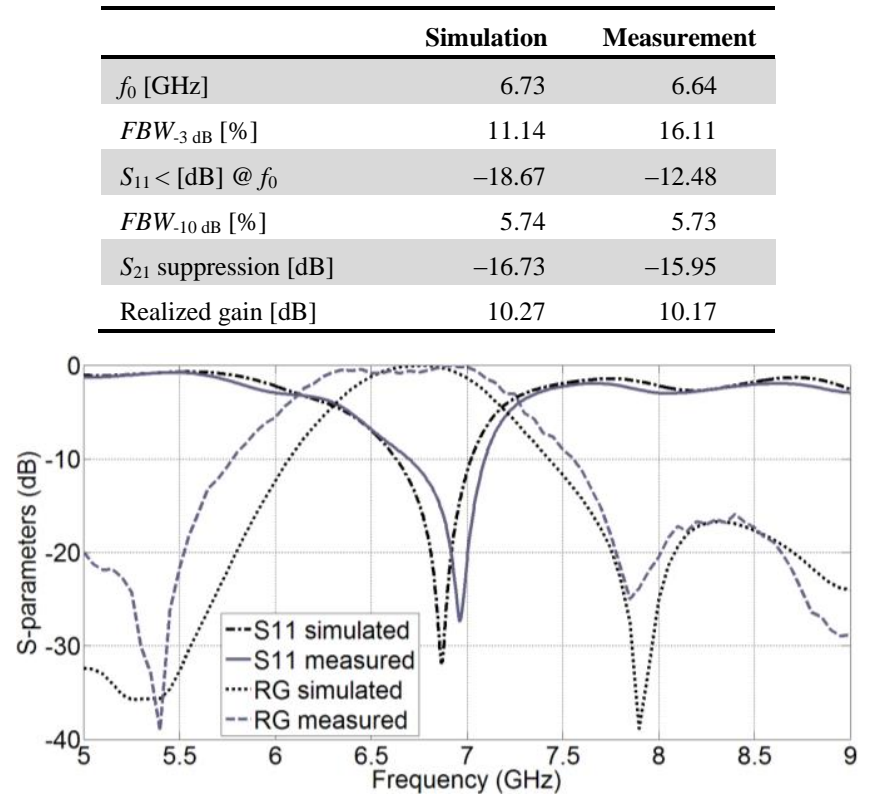

Fig. 10. Comparison of the simulated and measured frequency responses of reflection coefficient $S_{11}$ and normalized realized gain RG; $f_{0}=6.8 \mathrm{GHz} ; F B W=10 \%$ and $S_{11}<-10 \mathrm{~dB}$.

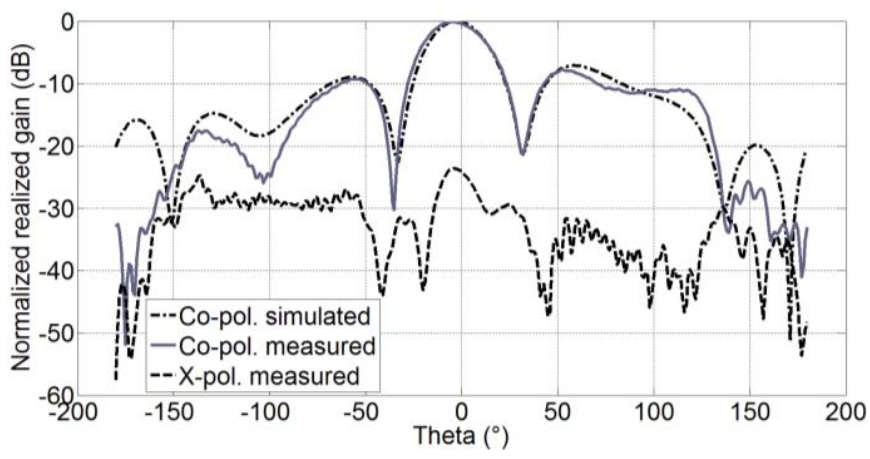

Fig. 11. Comparison of simulated and measured radiation patterns (co-polar and cross-polar components) in the E-plane at frequency $6.9 \mathrm{GHz}$.

in the E-plane (co- and cross-polarization components) are shown in Fig. 11. The deviation in the main lobe direction

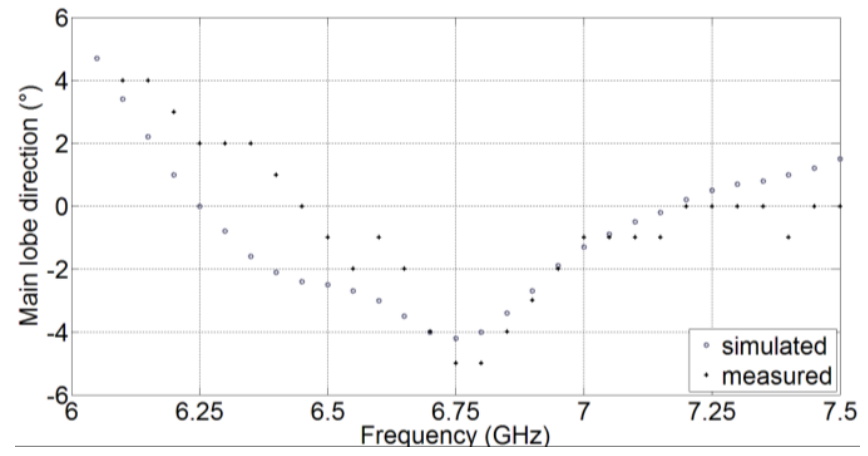

Fig. 12. Comparison of the simulated and measured frequency response of the main lobe direction of the filtenna over the operation band; $f_{0}=6.8 \mathrm{GHz} ; F B W=10 \%$ and $S_{11}<-10 \mathrm{~dB}$.
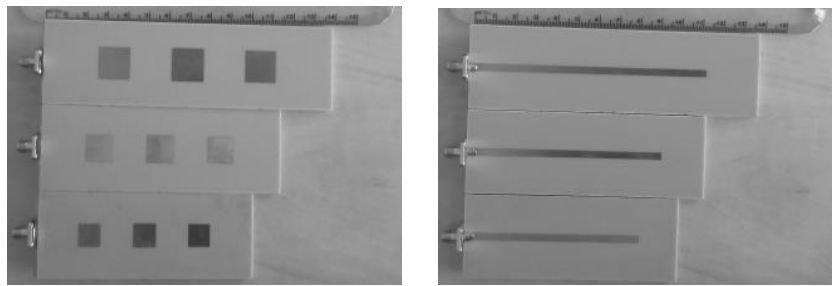

Fig. 13. Top (left) and bottom (right) layers of the manufactured three-element filtennas designed for the central frequency $4.8 \mathrm{GHz}$ (top); $5.8 \mathrm{GHz}$ (center) and $6.8 \mathrm{GHz}$ (bottom).

from the normal to the substrate is depicted in Fig. 12; the maximum deviation is $4.2^{\circ}$ for simulation and $5^{\circ}$ for measurement.

Obviously, simulations are in agreement with measurements. Deviations are caused by manufacturing: the filtering array consists of two dielectric substrates and is sensitive to the accuracy of assembly.

In Fig. 13, the top layers of the fabricated test filtennas are shown on the left; and the corresponding bottom layers on the right.

\section{CONCLUSION}

In this paper, we present the methodology for the design of third-order patch filtennas with aperture excitation. The methodology combines the filter design approach and the antenna design approach exploiting the equivalent circuit of the third-order filtering antenna array. The presented filtenna design procedure has not been published yet.

The filtenna design procedure was implemented in MATLAB. Outputs of the script sufficiently correspond with the full-wave model of the filtenna. Thanks to the script, we can save CPU time during the design of filtering arrays. The MATLAB script produces comprehensive results within 2 minutes on a conventional PC.

Prescribed center frequency, fractional bandwidth and magnitude of reflection coefficient are objectives of the design. The center frequency was varied and validated from $4.80 \mathrm{GHz}$ to $6.80 \mathrm{GHz}$, the fractional bandwidth was changed and verified from $7 \%$ to $13 \%$, and the magnitude of reflection coefficient was altered and validated from $-10 \mathrm{~dB}$ to $-20 \mathrm{~dB}$.

The frequency responses of the reflection coefficient and the frequency response of the realized gain were shaped using 
new $g_{i}$ coefficients which substituted the conventional $g_{i}$ coefficients of conventional approximations of filter characteristics (Chebyshev, Bessel, Butterworth). New $g_{i}$ coefficients were included into the filter design approach which is an integral part of the design methodology of filtering antenna arrays.

Two test examples were presented to illustrate the functionality of the developed design methodology. Then, corresponding samples of these filtering antennas were manufactured and measured. The measured data was in good agreement with the simulated ones. Small deviations were caused by manufacturing inaccuracies.

At present, the described synthesis of filtennas is generalized for a higher number of antenna elements. Moreover, tuning-space mapping is going to be used in combination with the presented equivalent circuit to improve efficiency of the design.

\section{ACKNOWLEDGMENT}

The presented research was financed by the Czech Ministry of Education within the National Sustainability Program under grant no. LO1401 and by the Czech Science Foundation under grant no. P102/12/1274. For computations and measurements, infrastructure of the SIX Center was used.

Contribution of Jordi Mateu was supported in part by the Spanish Government (grants TEC-2012-13897-C03-01 and MAT2011-29269-C03-02), and partly by the Cluster of Application and Technology Research in Europe on Nanoelectronics (CATRENE) under the project CORTIF CA116 (Coexistence of radio frequency transmission in the future).

\section{REFERENCES}

[1] Ch. Yu, and W. Hong, "37-38 GHz substrate integrated filtenna for wireless communication application", in Microwave and Optical Technology Letters, vol. 54, no. 2, pp. 346-351, Feb. 2012.

[2] S. Yu, W. Hong, Ch. Yu, H. Tang, J. Chen, and Z. Kuai, "Integrated millimeter wave filtenna for Q-LINKPAN application", Proceeding of the $6^{\text {th }}$ European Conference on Antennas and Propagation (EUCAP), Prague, Czech Republic, 26-30 March 2012, pp 1333-1336.

[3] Ch. Yu, W. Hong, Z. Kuai, and H. Wang, "Ku-band linearly polarized omnidirectional planar filtenna", in IEEE Antennas and Wireless Propagation Letters, vol. 11, pp. 310-313, March 2012.

[4] Y. Tawk, J. Costantine, and C. G. Christodoulou, "A varactor-based reconfigurable filtenna", in IEEE Antennas and Wireless Propagation Letters, vol. 11, pp. 716-719, June 2012.

[5] Y. Tawk, M. E. Zamudio, J. Costantine, and C. G. Christodoulou, "A cognitive radio reconfigurable "filtenna", Proceeding of the $6^{\text {th }}$ European Conference on Antennas and Propagation (EUCAP), Prague, Czech Republic, 26-30 March 2012, pp 3565-3568.

[6] M. Zamudio, Y. Tawk, J. Costantine, J. Kim, and C. G. Christodoulou, "Integrated cognitive radio antenna using reconfigurable band pass filters", Proceeding of the $5^{\text {th }}$ European Conference on Antennas and Propagation (EUCAP), Rome, Italy, 11-15 April 2011, pp.2108-2112.

[7] W. J. Wu, Y. Z. Yin, S. L. Zuo, Z. Y. Zhang, and J. J. Xie, "A new compact filter-antenna for modern wireless communication systems", in IEEE Antennas and Wireless Propagation Letters, vol. 10, pp. 11311134, Oct. 2011.

[8] S. J. Chung, and H. N. Wang, "Compact multi-function antennas designed using filter synthesis technique", Proceeding of the $42^{\text {nd }}$ European Microwave Conference (EuMC), Amsterdam, The Netherlands, Oct. 29 2012-Nov. 1 2012, pp. 1331-1334.
[9] Ch. T. Chuang, and S. J. Chung, "New printed filtering antenna with selectivity enhancement", Proceeding of the $39^{\text {th }}$ European Microwave Conference (EuMC 2009), Rome, Italy, Sept. 29 2009-Oct. 12009 , pp.747-750.

[10] Ch. T. Chuang, and S. J. Chung, "A new compact filtering antenna using defected ground resonator", Proceeding of the 2010 Asia-Pacific Microwave Conference (APMC), Yokohama, Japan, 7-10 Dec. 2010, pp.1003-1006.

[11] Ch. T. Chuang, and S. J. Chung, "A compact printed filtering antenna using a ground-intruded coupled line resonator", in IEEE Transactions on Antennas and Propagation, vol. 59, no. 10, pp. 3630-3637, Oct. 2011.

[12] Ch.-K. Lin, and S. J. Chung, "A compact simple structured filtering antenna utilizing filter synthesis technique", Proceeding of the 2010 Asia-Pacific Microwave Conference (APMC), Yokohama, Japan, 7-10 Dec. 2010, pp. 1573-1576.

[13] Ch. T. Chuang, and S. J. Chung, "Synthesis and design of a new printed filtering antenna", in IEEE Transactions on Antennas and Propagation, vol. 59, no. 3, pp. 1036-1042, March 2011.

[14] J. Verdu, J. Perruisseau-Carrier, C. Collado, J. Mateu, and A. Hueltes, "Microstrip patch antenna integration on a bandpass filter topology", Proceeding of the $12^{\text {th }}$ Mediterranean Microwave Symposium (MMS2012), Istanbul, Turkey, Sep. 2012.

[15] Ch. K. Lin, and S. J. Chung, "A compact filtering microstrip antenna with quasi-elliptic broadside antenna gain response", in IEEE Antennas and Wireless Propagation Letters, vol. 10, pp. 381-384, April 2011.

[16] Ch. K. Lin, and S. J. Chung, "A filtering microstrip antenna array”, in IEEE Transactions on Microwave Theory and Techniques, vol. 59, no. 11, pp. 2856-2863, Nov. 2011.

[17] M. Kufa, Z. Raida, and J. Mateu, "Equivalent circuits of planar filtering antennas fed by apertures", Proceeding of the $20^{\text {th }}$ International Conference on Microwaves, Radar, and Wireless Communication (MIKON), Gdansk, Poland, 16-18 June 2014, pp. 1-4.

[18] J. S. Hong, and M. Lancaster, Microstrip filters for RF/microwave applications. New York: John Wiley, 2001. ISBN 04-713-8877-7.

[19] D. M. Pozar, Microwave engineering, 3rd ed. Hoboken: John Wiley, 2005. ISBN 978-0-471-44878-5.

[20] R. Garg, Microstrip antenna design handbook. Boston: Artech House, 2001. ISBN 08-900-6513-6.

[21] C. A. Balanis, Antenna theory: analysis and design. 3rd ed. Hoboken: Wiley-Interscience, 2005. ISBN 978-0-471-66782-7.

[22] C. A. Balanis, Modern antenna handbook. Hoboken, NJ: Wiley, 2008. ISBN 04-700-3634-6.

[23] T. A. Milligan, Modern antenna design. 2nd ed. Hoboken: IEEE Press; John Wiley, 2005. ISBN 04-714-5776-0.

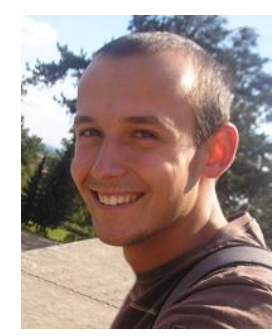

Martin Kufa was born in 1988 in Chlumec nad Cidlinou, Czech Republic. In 2012, he received his MSc. degree from the Faculty of Electrical Engineering and Communication (FEEC), Brno University of Technology (BUT). In 2015, he received his Ph.D. degree from the FEEC BUT. His Ph.D. thesis was focused on "Numerical synthesis of electromagnetic structures". His research interests include planar fractal filter design, design of antenna arrays and filtering antenna arrays. From $25^{\text {th }}$ of November to $20^{\text {th }}$ of December 2013 and from $5^{\text {th }}$ of October to $31^{\text {st }}$ of October 2014, he was in the Polytechnic University of Catalonia, Spain, and working on equivalent circuit and numerical synthesis of filtering antenna array. He was chairman of the IEEE Sudent Branch of the Brno University of Techlonoly in 
2014 and in 2015, he was Vice-chairman of the IEEE Student Branch of the Brno University of Technology.

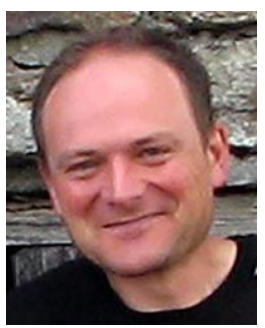

Zbynek Raida has graduated at Brno University of Technology, Faculty of Electrical Engineering and Communication (FEEC BUT). Since 1993, he has been with the Department of Radio Electronics FEEC BUT, now as a Professor. In 1996 and 1997, he was with the Laboratoire de Hyperfrequences, Universite Catholique de Louvain, Belgium, working on variational methods of numerical analysis of electromagnetic structures.

Zbynek Raida has been working on numerical modeling and optimization of electromagnetic structures, exploitation of artificial neural networks for solving electromagnetic issues, and the design of antennas. Zbynek Raida is a member of IEEE MTT Society.

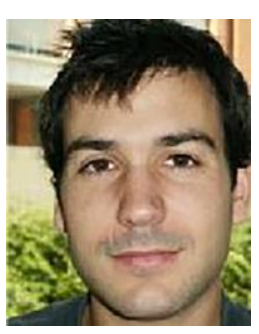

Jordi Mateu is Associate Professor in the Signal Theory and Communications Department (TSC) at the Universitat Politècnica de Catalunya (UPC), since March 2009, where he joined as a Ramon y Cajal Research Fellow in November 2006. $\mathrm{He}$ is also external Associate Researcher at Centre Tecnològic de Telecomunicacions de Catalunya (CTTC), since 2007. He received the Telecommunication Engineering and Ph.D. degrees from the UPC, Barcelona, in 1999 and 2003, respectively.

Prior to that, in summer 1999, after graduation, he held a trainee engineer position at Investment Technology department, Gillette, UK. In 2000, he joint TSC department to pursuit Ph.D. studies. From May to August 2001, he was
Visiting Researcher with Superconductor Technologies Inc., Santa Barbara CA. In October 2002 he joined CTTC as Research Associate and later as Coordinator of Communication Subsystems Area, until August 2005. During that period he was also Part-time Assistant professor at Universitat Autònoma de Barcelona. In September 2005, he joined the National Institute of Standards and Technology (NIST), Boulder, CO, as Fulbright Research Fellow until October 2006. Since then he has held several Guest Research Appointments at NIST (summer 2007, summer 2008, April 2009, January 2015 and May 2015). In July 2006, he was visiting Researcher at Lincoln Lab., MIT. He authored or coauthored over 50 papers in international journal, more than 60 contributions in international conferences, three book chapter and holds two patents. He has collaborated and lead several research project for national and international public and private organizations and companies.

Dr. Mateu was the recipient of the 2004 Prize for the best doctoral thesis in Fundamental and Basic Technologies for Information and Communications by COIT (Colegio Oficial de Ingenieros de Telecomunicación) and AEIT (Asociación Española de Ingenieros de Telecomunicación). He was also the recipient of a Postdoctoral Fulbright Research Fellowship and an Occasional Lecturer Award from CIES (Council of International Exchange of Students). He was granted with a Ramón y Cajal Contract (2005-2010), being ranked second at National level, in the area of Electrical and Communication Technologies, a National Program for promoting outstanding Young researchers. He was granted by the Spanish government to pursuit with his $\mathrm{PhD}$ studies (2000-2003). From February 2011 to June 2012 he was ViceDean at Telecommunication and Aerospace Engineering School at UPC, in charge of academic affairs. He is IEEE Senior member. He is reviewer of several journals and international conferences. 